\title{
Welding Robot Control Based on Network
}

\author{
Wen-ming Zhang, Zheng-kai Xiong, Yan-fang Wang \\ Department of Mechanical Engineering, Shenyang University, Shenyang, 110044, China \\ Lingyuan Iron and Steel Group Company, Chaoyang, 122500, China
}

Keywords: robot, Intelligent welding, PLC, Field bus.

\begin{abstract}
This paper introduces principle of wielding robots trajectory controlling and network control system based on PLC and field bus. The key technology including: motor control based on servo-control system technology and parameters real-time control technology. The introduction of principle of PLC and field-bus controlling let the problems of information transmission and coordination control between automatic lines solve.
\end{abstract}

\section{Introduction}

The robot is a set of mechanical design, servo control, trajectory planning, control algorithm, sensor technology in an electromechanical integration equipment. The robot has very strict requirements for precise trajectory control, especially for a welding robot which absolute link with the track precision and welding quality. With the development of welding robot workstation becomes more and more intelligent, automatic programming technology of welding tasks and dynamic real-time feedback technology of welding quality is also very important.

\section{Robot trajectory control}

Rotational motion of each joint robot ultimately comes down to the respective axis AC servo motor. Industrial robots develop coordinated multi-axis path control problems is the key to the robot motion control. Operator via teach pendant or off-line programming, the programming instructions or software instructions needed to complete the task the robot controller, after evacuation axis computer-level language compiler computer algorithm for motion processing, generate and coordinate transformation trajectory.[1]After processing the signals generated by the drive to complete the three-phase drive current pulse width modulation, and then coordinated control of each joint motor rotation, to achieve the end of the robot wrist, direct implementation of the work required by the operator in accordance with the requirements of the tool center point hungry trajectory. Joint motor control of robot is shown in Figure 1. Most of the industrial robot controller using two computer architecture, computer within the dashed box is the first stage.

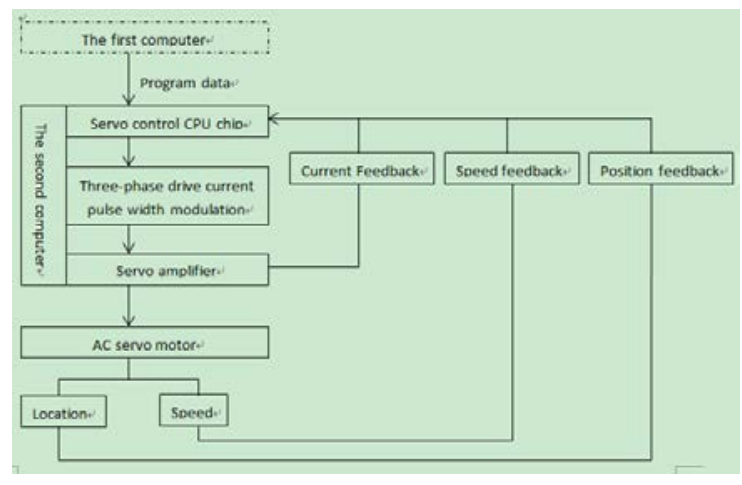

Fig1. Robot joint motor control schematics

\section{The first computer}

A computer task planning and management of the robot work process all the information and 
control all of its action. Robots at teaching status, accepted teaching system sent each teaching position and attitude information, movement parameters and process parameters, and by calculating the conversion teach each point (joint) to Cartesian coordinate values values exist into the computer's memory. Robots run automatically when removed from memory by point coordinate values its position and attitude, according to beat a certain time (also known as the sampling period) for circular or linear interpolation of its operation, calculates the position and orientation coordinates of interpolation points value, which is generated path planning. Then converted point by point coordinate values of the position and attitude of each interpolation point of a joint coordinate values, distributed to each joint. This is the first level of the computer trajectory planning process.

\section{The second computer}

Also known as the axis computer, servo amplifiers and CPU module for the main components within the servo motor connected to the robot arm, and its mission is to conduct a closed-loop servo motor control. After it receives the first stage of the joint computer sent the next is expected to reach the position and attitude, uniform segmentation, in order to be more smooth trajectory. Then fine step-by-point expectations of each joint to the drive motor, the simultaneous detection photoelectric encoder signal until its exact place.

With high-performance microprocessor (MCU), digital signal processors (DSP) development, digital servo control technology has become the mainstream of industrial servo system, CPU program by pulse width modulation to convert the data into the servo drive signals to the servo amplifier, then control the servo motor is rotated in accordance with a given signal, the encoder in real time through the speed control. Typically by the CPU module further includes different types of I/O modules are connected to achieve the role of other external devices or robots communicate with.

\section{Robot trajectory control}

Robot control computer only to ensure precise movement of the robot, but also is scalable to control peripheral devices to ensure the implementation of the welding process. Welding controller is usually caused by CPU, EPROM and some peripheral interface chips minimum control system, not only to complete the welding parameters input and real-time changes with the robot trajectory based on predetermined welding machine welding procedures, and timely and accurate welding system faultdiagnosis, and to achieve interactive communication with the robot control computer and the teaching box.

\section{Robot control peripherals}

Welding robot workstation in addition to controlling the movement of the robot hand, the need to control the actions of the external device is turned off and safety, etc., a complete set of arc welding robot system, which includes a robot manipulator, control systems, welding equipment, welding folder holding device and the like.

Robot control system and communication signal for all devices have a digital signal and analog signal. Control cabinet and peripherals analog signal linked to a welding power source, wire feeder and operating machines (including fixtures, displacement, etc.). These devices need to preset parameters via the control system, usually through D / A DAC given reference voltage, the controller and the welding power source and wire feeder mains are generally required to have optical isolation, the control system for operating the machine servo motor control the robot servo control motors require similar, usually double servo loop weld in place to ensure the accuracy of the workpiece in place with the robot accuracy equal.

\section{Intelligent welding quality control}

Welding process parameters on the quality of welding has the most direct impact, so crucial to determine the exact parameters. Typically welding parameters are automatically generated by the welding parameters planning controller to weld seam spatial information data such as location, joint form and shape required to enter into the argument programming model is complete, the programming model is usually neural network theory, fuzzy control theory and experts integrated use of [2], planning parameters typically include welding current, welding voltage, welding torch 
and torch working angle velocity system theory three models. However, individual, running speed and working angle torch welding equipment is not an independent decision by the robot control velocity and joint posture respectively. It must in order to ensure the quality of welding by these organic complexes welding parameters, thus allowing the robot motion control and planning welding parameters closely coupled to form a unified whole joint planning to generate more accurate welding parameters to ensure the welding quality.

Dynamic real-time information feedback welding process is the key to welding quality control, intelligent sensor feedback mainly refers to the process of welding puddle size, penetration, and Tuen arc forming behavior parameters online testing, in order to achieve real-time control of welding quality. Visual seam tracking and weld pool state feedback is the core and foundation of welding robot sensing system, in order to obtain a three-dimensional contour of the weld joints and overcome interference arc welding process, the robot recognizes seam tracking technology is generally used laser structured light and other active vision approach weldment feature extraction, to correctly guide the robot along the actual welding torch terminal to complete the desired trajectory. Due to the complexity of the welding process disturbances, as well as the role of a large number of strong nonlinear uncertainties, making detection of the welding process to become a reliable and practical attention problems. From the pool of dynamic change and penetration feature detection perspective, is better at present computer vision technology, temperature field measurement, the pool incentive oscillation arc sensing method for real-time control.

\section{Robot trajectory control}

Robotic welding stations often used as a production line from the station, and a larger number of scattered locations, production automation all robots must be connected to a form of unified network management coordination and control. PLC as the control center in real time via the fieldbus, field welding equipment and welding robots to communicate and connect to other devices such as human-machine interface (console and PC), various safety relays, safety equipment terminal (such as laser scanners, safety grating, safety gates etc.) to form an industrial automation and control networks and for network terminal problems, timely treatment. This network can be connected through a variety of communication lines with the host computer, to form a large scale, strong function and high reliability integrated network control system (Figure 2) of the robot production line monitoring, diagnosis and management [3]. Fieldbus and network terminal module will be collected signals to the PLC, PLC logic according to a pre-programmed procedures make the appropriate treatment of the received signal and sends the results to the relevant system variables to achieve an orderly and stable continuous operation.

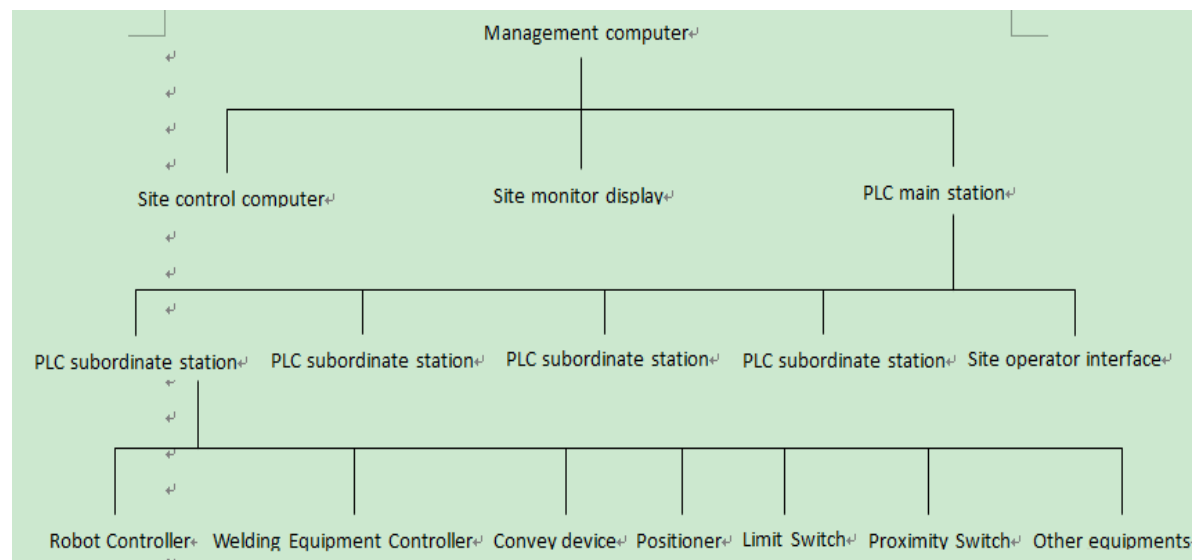

Fig2. Welding robot network control chart

\section{PLC control}

Programming Logic Controller (PLC) hardware can include: central control processing unit CPU, memory; input and output interfaces; power and programmer. PLC programmable logic control 
instructions is the most basic of the most common commands, is a basic component of the ladder and statements constitute the table. The basic logic control instructions are usually pointing logic instructions, timers and counters command instruction. Logic control procedures commonly used class ladder programming, which relay circuit and control logic ladder same concept to express the relationship between the system of all the action. If you use a graphics programmer (LCD or CRT), then draw the equivalent of the preparation of a ladder program, can be fed directly into the programmable controller ladder. For simple programmer, you often have to go through the conversion process mnemonic program before it can be entered into the programmable controller.

Network composed of PLC control system is realized on the basis of the whole, but also to all those who collect data specific control, measurement, processing and transmission, PLC control field hand coordination process sequence in accordance with the requirements of the various devices work; on the other hand it accepts programming device configuration information to the upper device sends real-time production information and device status information for the upper device statistics, diagnostics and display. PLC and robot control individually programmed production line, so real-time communication between PLC and robot is very important. Question and answer type of network communication via serial communication interface to send commands the robot controller: Notify ready robot welding, robotic welding, welding robot stops (fails). Robot feedback to the PLC instruction is robotic welding posture, the robot operating status, PLC communications and information based on the next step the robot feedback. PLC programs need to issue a call to signal the robot according to the production line running, according to a corresponding robot built-in controller for signal processing program, call the predefined Teach program modules.

\section{Fieldbus network}

Fieldbus is used in the production of a scene between field devices, two-way serial between field devices and control devices, multi-junction point digital communications technology. Information transfer problem between these field of digital communication and control equipment and advanced control system is mainly to solve the industrial field of intelligent devices, controllers, actuators and other field devices. Usually twisted pair RS-485 technology as a communication cable for data exchange, the scattered field devices (intelligent, with communication interfaces such as RS-485 serial interface) and the computer is connected to a network remote monitoring system, data transmission exchange of information, the formation of various meet the practical needs of fieldbus control system (FCS). The system is both an open communication network, but also a fully distributed control system, it distributed control system (DCS) the biggest difference is that the common field bus or a dedicated microprocessor into traditional field measurement and control equipment, the site can be completed by the middle of the controller to complete the low-level control function, making the automatic control system also joined the ranks of information networks.

Robot welding production line control using a three-tier network architecture for networking the entire production line. The first layer of Internet-based PC management and WAN, for statistical departments of management and information; the second layer is an Ethernet-based PC (industrial PC) and various production lines of the main controller network layer, with to monitor the situation; the third layer of the network field bus master controller and execution units based at the bottom of the industrial network systems, directly connected to the scene of a variety of devices (such as I / O devices, sensors, transmitters, frequency and drivers, etc.) in real-time on-site control, signal feedback.

\section{Conclusions}

Welding robot design parameters typically include the number of degrees of freedom, the scope of work, load capacity, welding speed and precision welding, the welding process requires not only accurate and consistent weld torch trajectory, but also to ensure proper gun gesture. Intelligent and self-programming related welding technology is a hot academic research, including welding mission planning, trajectory tracking control, sensor systems, process control and other aspects of the model as well as intelligent, intelligent welding means that in the work process, through 
integration enables automatic identification of the weld, weld path planning, seam tracking and constant pressure control various sensors and closed-loop control system. Intelligent control also includes all except by its own robot control system controls autonomous operation, it must also accept remote control programmable controller and on-line monitoring system via the network control system to ensure the welding robots and other automated equipment production line synchronous operation.

\section{References}

[1] Sun Hanqing, Wu Haibo. principle articulated robot and maintenance [M] Beijing: National Defense Industry Press, 2013.

[2] Bi Xiaofeng. robotic arc welding robot teaching principles and programming [J] welding, 2009,39 (4): 83-86.

[3] You Yangyang. PLC control system auto white car sound welding production line design and implementation of [D] Xi'an: Chang'an University, 2009. 